\title{
Energy Spectrum of the Recurrent Variation of Galactic Cosmic Rays During the Solar Minimum of Cycles 23/24
}

\author{
Agnieszka Gil $^{1}$ (D) Michael V. Alania $^{1,2}$
}

Received: 24 February 2016 / Accepted: 23 May 2016 / Published online: 21 July 2016

(C) The Author(s) 2016. This article is published with open access at Springerlink.com

\begin{abstract}
The Sun during the recent epoch of solar activity operated in a different way than during the last 60 years, being less active. We study temporal changes of the energy spectrum of the first three harmonics of the 27-day variation of the galactic cosmic rays (GCR) intensity during the unusual, recent solar minimum, between Solar Cycles 23 and 24 (SC 23/24) and compare with four previous minima. We show that the energy spectrum of the amplitudes of the recurrent variation of the GCR intensity is hard in the maximum epochs and is soft in the minimum epochs during Solar Cycles 20-24, but with peculiarities during the Solar Minimum 23/24. In particular, while the energy/rigidity spectrum of the amplitudes of the first harmonic of the recurrent variation of the GCR intensity behaves practically the same as for previous epochs, the energy/rigidity spectrum of the amplitudes of the second and the third harmonics demonstrates a pronounced softening. We attribute this phenomenon to the decrease of the extension of the heliosphere caused by the decrease of the solar-wind dynamic pressure during the unusual Solar Minimum 23/24.
\end{abstract}

Keywords Solar activity · Energy spectrum · Recurrence of galactic cosmic rays intensity

\section{Introduction}

Galactic cosmic rays are highly energetic nuclei of extra-solar origin. When they are propagating through the heliosphere, they are partially modulated by the solar wind and heliospheric magnetic field (HMF). Moreover, the heliosphere changes over time, and those changes influence the GCR stream on different time scales (e.g. Kudela, 2013; Yeeram et al., 2014). The recurrent variation of the galactic cosmic-ray intensity was described for the

\section{A. Gil}

gila@uph.edu.pl

M.V. Alania

alania@uph.edu.pl

1 Institute of Mathematics and Physics, Siedlce University, Siedlce, Poland

2 Institute of Geophysics, Tbilisi State University, Tbilisi, Georgia 
first time in the 1930s (Forbush, 1938) and is still of interest. Among recent publications devoted to this issue one can mention, e.g., Richardson (2004); Dunzlaff et al. (2008); Gil, Modzelewska, and Alania (2012); Guo and Florinski (2014). However, changes of the amplitudes of the recurrent variation of the GCR intensity versus the rigidity $\left[R=\sqrt{T^{2}+2 T T_{\mathrm{r}}}\right.$, where $T$ is kinetic energy and $T_{\mathrm{r}}=0.938 \mathrm{GeV}$ is a proton's rest-mass energy] were studied very rarely until the work of Gil and Alania (2008). Gil and Alania (2010, 2013b) demonstrated that during the period 1965-2002 the rigidity spectrum of the recurrent variation of the GCR intensity is hard in the maximum epochs, and it is soft in the minimum epochs of solar activity (SA). We relate this phenomenon to the changes of the effective size $[L]$ of the modulation area of the recurrent variation of the GCR intensity in different epochs of SA. The effective size $[L]$ of the modulation region of the GCR recurrence is smaller in the minimum epochs than in the maximum epochs. We base this explanation on the relation of the GCR particle's rigidity $[R]$ with the product of $B \rho(R \propto B \rho)$, where $B$ is the strength of the regular part of the HMF in the vicinity of heliolongitudinal asymmetry of the solar wind (being the source of the recurrent variation of the GCR intensity), and $\rho$ is the cosmic-ray particle's Larmor radius. When other conditions are equal, the larger are $\rho$ and $B$, the higher-energy are the cosmic-ray particles that are involved in the modulation, and the energy/rigidity spectrum of the recurrent variation of the GCR intensity, consequently, becomes harder.

The maximum epoch of the current SC 24 was about $50 \%$ weaker than the maxima of the four previous SCs (e.g. Bazilevskaya et al., 2014; Balogh et al., 2014), similarly as the unusual Solar Minimum 23/24 (e.g. Dikpati, 2013) was weaker comparing with the previous minima. The sunspot number (SSN) is treated as a key indicator of solar activity. During SC 23 the lowest value of smoothed SSN was 1.7 in December 2008, while the lowest SSN in SC 22 was 12.6 (e.g. Dikpati, 2013). McComas et al. (2008) indicated a huge drop $(\approx 36 \%)$ in the average strength of the HMF. The lowest monthly value of HMF strength during the last minimum was $3.5 \mathrm{nT}$ in April 2009. Sheeley (2010) concluded that the polar fields were weaker than they have been in the last 100 years. According to Hoeksema (2010) that behavior could signal a weaker maximum of Solar Cycle 24, which indeed took place (e.g. Balogh et al., 2014). These low values of the polar field throughout the last solar cycle caused a much slower decreasing of the tilt angle of the heliospheric current sheet (up to 2008), in contrast to Cycles 21 and 22. The average solar-wind speed (SWs) during this $23 / 24$ solar minimum was $388 \mathrm{~km} \mathrm{~s}^{-1}$, being $8 \%$ slower than in the previous minimum, and $15 \%$ slower than earlier ones (e.g. Jian, Russell, and Luhmann, 2011). This might be caused by the fact that the polar coronal holes were smaller than in previous cycles. Abramenko et al. (2010) reported that the area occupied by the near-equatorial coronal holes $\left( \pm 40^{\circ}\right)$ was larger during the Minimum 23/24 in comparison with the previous solar minimum. Kirk et al. (2009) stated that the north and south polar-hole areas were reduced by $\approx 15 \%$ in 2007 compared with 1996. Mason et al. (2009) found that during the recent Minimum 23/24 in comparison with the previous one $(22 / 23)$ there were many more co-rotating interaction regions observed, connected with the existence of more high-speed streams of solar wind. Moreover, a low solar-wind dynamic pressure, $\approx 40 \%$ in comparison with the previous minimum, was observed (Mewaldt et al., 2010) and this was caused by the drop in the average proton density, which was $\approx 5.5 \mathrm{~cm}^{-3}$, being less dense in comparison with the previous three minima by $\approx 70 \%$ (Jian, Russell, and Luhmann, 2011). All of the phenomena and processes mentioned caused diminishing of the solar-modulation influence on GCR intensity and its variations.

McComas et al. (2008) recognized a shrinkage of the size of the heliosphere (caused by the drop of the solar-wind dynamic pressure), which should result in an increase in the GCR 
intensity at $1 \mathrm{AU}$ (Mewaldt et al., 2010). Indeed, the GCR intensity values measured by neutron monitors were the highest ever recorded (e.g. Moraal and Stoker, 2010; Oh et al., 2013). In 2007, neutron monitors (NMs) recorded values last measured during the last $A<0$ solar minimum, 1985-1987, and the values continued to grow, exceeding the previous levels by a few percent, throughout the end of 2009. Stratospheric balloon measurements showed an even higher increase: $\approx 20 \%$ (Stozhkov et al., 2009). The Payload for Antimatter Matter Exploration and Light-nuclei Astrophysics (PAMELA) experiment observed that from mid-2006 to the end of 2009 the cosmic-ray spectra became softer (up to the intensity of 2.7 particles $\left(\mathrm{m}^{2} \mathrm{~s} \mathrm{sr} \mathrm{MeV}^{-1}\right.$ (e.g. Vos and Potgieter, 2015) since gradually more protons with lower energy were able to reach the Earth (Potgieter et al., 2014). Mewaldt et al. (2010) found $\approx 44 \%$ growth of the parallel mean free path of GCR particles $\left[\lambda_{\|}\right]$from the minimum in 1997 to 2009 and its rapid reduction in 2010. Cliver, Richardson, and Ling (2013) stated that particle drifts were not the main processes in the recent minimum, but the GCR modulation was determined by diffusion and convection, contradicting the results obtained by Alania, Modzelewska, and Wawrzynczak (2014), i.e. that a drift related to the delay time between tilt angles of heliospheric current sheet and cosmic-ray intensity was an important reason for an increase of cosmic-ray intensity in 2009. Potgieter et al. (2014) indicated that diffusion and particle-drift contributions were both $\approx 50 \%$ for the GCR protons observed at the Earth. The average mass and kinetic energy of coronal mass ejections were around five times lower in comparison with the previous minimum (Vourlidas et al., 2010), diminishing one of the barriers for entrance of GCR particles into the inner heliosphere (Mewaldt, 2013). Zhao et al. (2014) also stated that a low magnetic turbulence, increasing the parallel diffusion and reducing the perpendicular diffusion in the polar direction, might be another phenomenon giving rise to the observed higher GCR intensity.

The amplitudes of a new type of quasi-periodicity of the GCR intensity, found using the amplitudes of the 27-day variation, called the three to four Carrington-rotation period (3-4 $\mathrm{CRP}$ ) recurrence during the last minimum epoch had similar values as in the previous minimum, much lower than during the solar minimum one Hale cycle earlier (Gil and Alania, 2013a). It was ascribed to the anomaly increasing the diffusion of cosmic rays (Moraal and Stoker, 2010) during the Minimum 23/24. Dunzlaff et al. (2008) reported, based on Ulysses observational data, that in SC 22 the decrease in the component of the recurrent GCR arising from the fast solar wind showed a visible maximum from $25^{\circ}$ to $40^{\circ}$ and beyond, but in SC 23 there was no such substantial change. The authors suggested that the absence of large, stable, coronal-hole structures in SC 23 might be a reason for this dissimilarity (e.g. Kirk et al., 2009). Guo and Florinski (2014) stated that the GCR recurrent-modulation effect of co-rotating interaction regions played a significant role. Gil, Modzelewska, and Alania (2012) reported that the average amplitude of the recurrent variation of the GCR intensity calculated for the mid-latitude NMs was as high as during the previous minimum epoch $1996(A>0)$ and much higher than during the minimum one Hale cycle earlier; but at the same time, the average amplitude of the recurrent periodicity of the GCR anisotropy was at the same level as in the previous negative-polarity cycle. Modzelewska and Alania (2013) showed that the recurrent variation of the GCR intensity was stable during 2007 2008 and evolved to longer periods (up to 33 - 36 days) during 2009. Aslam and Badruddin (2015) showed, using wavelet analysis, that the recurrent variation during the last minimum remained steady compared with the four previous minima.

Because of all of those facts, it is important to check that the strength of the solar activity influenced the spectrum of the recurrence of the GCR intensity. Hence, the main aim of this article is to study the behavior of the rigidity spectrum of the recurrent variation of the galactic cosmic rays during the recent epochs of SA, particularly in the period covering the last solar-minimum and solar-maximum epochs. 


\section{Data and Mathematical Background}

We present the computed rigidity spectrum of the amplitudes of the first three harmonics of the recurrent variation of the GCR intensity during the maximum of SC 23 up to the ongoing SC 24 . For this purpose we used daily data from eight neutron monitors from a worldwide network, with different cut-off rigidities, in the period 01 January 2000 to 31 December 2014. Table 1 presents details, such as location and effective cut-off rigidity, of the neutron monitor (NM) stations used in this article.

Using harmonic analysis (e.g. Kincaid and Cheney, 2002) we computed the amplitudes of the first three harmonics of the 27-day variation of the GCR intensity by means of daily data from all NMs for each consecutive solar rotation during the period considered. For this purpose we used the formula

$$
x(k \Delta t)=\frac{a_{0}}{2}+\sum_{n=1}^{K / 2}\left[a_{n} \cos \left(\frac{2 \pi}{T} n k \Delta t\right)+b_{n} \sin \left(\frac{2 \pi}{T} n k \Delta t\right)\right],
$$

where $a_{n}=\frac{2}{K} \sum_{k=1}^{K} x(k \Delta t) \cos \frac{2 \pi k n}{K}, b_{n}=\frac{2}{K} \sum_{k=1}^{K} x(k \Delta t) \sin \frac{2 \pi k n}{K}, K=27$ days, and $A=$ $\sqrt{a_{j}^{2}+b_{j}^{2}}, j \in\{1,2,3\}$ is the amplitude of the $j$ th harmonic of the 27-day variation of the GCR intensity.

The variability of the primary spectrum of the GCR flux in the heliosphere can be defined by (e.g. Dorman, 2004)

$$
\left[\frac{\delta D(R)}{D(R)}\right]_{i}=\frac{D_{i}(R)-D_{0}(R)}{D_{0}(R)},
$$

where $D_{i}(R)$ means the primary spectrum of GCR during the $i$ th rotation and $D_{0}(R)$ the primary spectrum for the considered period. A typical procedure of reconstructing the primary cosmic-ray characteristics from NMs measurements is by the usage of the neutron-monitor yield function $Y(R, h)$ [in units of $\mathrm{m}^{2} \mathrm{sr}$ ] dependent on the primary particle rigidity/energy and observational level $h$ (e.g. Dorman, 1974; Clem and Dorman, 2000; Mishev, Usoskin, and Kovaltsov, 2013):

$$
N\left(R_{c}, h, t\right)=\int_{R_{c}}^{\infty} Y(R, h) D(R, t) \mathrm{d} R,
$$

where $N$ means count rates of specific NM, $D(R, t)$ primary spectrum (in units of particles $\left.\left(\mathrm{m}^{2} \mathrm{srs} \mathrm{GeV} \mathrm{nucleon}^{-1}\right)^{-1}\right)$ of GCR at the orbit of the Earth depending on rigid-

Table 1 Neutron monitors used in order to calculate the rigidity spectrum of the first three harmonics of the recurrence of the GCR intensity.

\begin{tabular}{llll}
\hline Neutron monitor & Geographical latitude and longitude & Effective vertical cut-off rigidity [GV] \\
\hline Apatity & $67.57^{\circ} \mathrm{N}$ & $33.4^{\circ} \mathrm{E}$ & 0.65 \\
Fort Smith & $60.02^{\circ} \mathrm{N}$ & $111.93^{\circ} \mathrm{W}$ & 0.3 \\
Jungfraujoch & $46.55^{\circ} \mathrm{N}$ & $7.98^{\circ} \mathrm{E}$ & 4.5 \\
Kiel & $54.34^{\circ} \mathrm{N}$ & $10.12^{\circ} \mathrm{E}$ & 2.36 \\
Moscow & $55.47^{\circ} \mathrm{N}$ & $37.32^{\circ} \mathrm{E}$ & 2.43 \\
Oulu & $65.05^{\circ} \mathrm{N}$ & $25.47^{\circ} \mathrm{E}$ & 0.8 \\
Potchefstroom & $26.42^{\circ} \mathrm{S}$ & $27.05^{\circ} \mathrm{E}$ & 6.94 \\
Rome & $41.86^{\circ} \mathrm{N}$ & $12.47^{\circ} \mathrm{E}$ & 6.27 \\
\hline
\end{tabular}


ity and time. Thus, the amplitudes of the recurrence of the GCR intensity for the $k$ th neutron monitor with characteristic cut-off rigidity $R_{\mathrm{c} k}$ can be calculated as follows (e.g. Dorman, 2004):

$$
\frac{\delta I_{i k}}{I_{i k}}=\frac{N_{i k}-N_{k 0}}{N_{k 0}}=\int_{R_{c k}}^{R_{\max }}\left[\frac{\delta D(R)}{D(R)}\right]_{i} \frac{D_{0}(R) Y\left(R, h_{k}\right)}{N_{k 0}} \mathrm{~d} R,
$$

where $[\delta D(R) / D(R)]_{i}$ means the rigidity spectrum for the $i$ th rotation. With the aid of the broadly used approximation, we define the rigidity spectrum of the recurrent variation as (e.g. Dorman, 1974; Yasue et al., 1982):

$$
\frac{\delta D(R)}{D(R)}= \begin{cases}a R^{-\gamma} & \text { for } R \leq R_{\max }, \\ 0 & \text { for } R>R_{\max },\end{cases}
$$

where $R_{\max }$ is a lower limit of the rigidity values above which the recurrence does not exist, in our article $R_{\max }=200 \mathrm{GV}$ (e.g. Alania and Wawrzynczak, 2012), $a$ is a constant, for $R_{0}$ being equal to $\delta D(R) / D(R)$, and $\gamma$ is the spectral index for the amplitudes of the harmonics of the 27-day variation of the GCR intensity. Using Equations (1) and (2) one can write

$$
\frac{\delta I_{i k}}{I_{i k}}=a_{k} \int_{R_{\mathrm{ck}}}^{R_{\max }} R^{-\gamma} Y\left(R, h_{k}\right) \mathrm{d} R .
$$

Thus

$$
a_{k}=\frac{\delta I_{i k}}{I_{i k}} / \int_{R_{\mathrm{ck}}}^{R_{\max }} R^{-\gamma} Y\left(R, h_{k}\right) \mathrm{d} R .
$$

Owing to the yield function, the 27-day variation amplitudes for each NM should be the same (to the accuracy available); hence in order to find spectral index $\gamma$ for a particular solar rotation one has to minimize the expression (using the least-square method):

$$
\sum_{k=1}^{\mathrm{NMs}}\left(a_{k}-a_{\mathrm{av}}\right)^{2}
$$

where $a_{\mathrm{av}}$ is an average amplitude of the 27-day variation of the GCR intensity for all NMs. Finally, values of

$$
\int_{R_{\mathrm{ck}}}^{R_{\max }} R^{-\gamma} Y\left(R, h_{k}\right) \mathrm{d} R
$$

were found for various $R_{\max } \in\langle 30,200\rangle \mathrm{GV}$ and $\gamma \in\langle 0,2\rangle$ by the Lagrange linear interpolation (e.g. Wawrzynczak and Alania, 2010).

To compare the behavior of the spectral index $\gamma$ of the 27-day variation of the GCR intensity during the ongoing SC 24 and especially the recent, unusual Solar Minimum 23/24 with its behavior during four previous SCs 20-23 we need to have continuous values of $\gamma$ for a long period. For this purpose, neutron monitors with substantially different cut-off rigidity, with well-established, continuous measurements over a long period, are essential. This requirement is reasonably well fulfilled by the Kiel and Rome NMs.

Using those NMs with the aid of the above-described method we compute values of the spectral index $\gamma$ of the first three harmonics of the recurrent variation of the GCR intensity for the period of 01 January 1964 to 31 December 2014. 


\section{Behavior of the Rigidity Spectrum of the 27-Day Variation of the GCR Intensity During Solar Cycles 23/24}

To study precisely the behavior of the energy/rigidity spectrum of the recurrent variation of the galactic cosmic-ray intensity during the current Solar Cycle 24 and the unusual Solar Minimum 23/24, we use eight various neutron monitors (Table 1) with considerably different cut-off rigidity during the period of 01 January 2000 to 31 December 2014. Figure 1 presents the results of the calculations. To emphasize the properties of the long-term changes of the rigidity spectra, we smoothed the values of the spectral index $[\gamma]$ with a running average over 39 solar rotations. The upper panel of Figure 1 shows that during the recent, unusual minimum epoch 23/24 the value of the spectral index $[\gamma]$ of the amplitudes of the first harmonic of the recurrent variation of the GCR intensity was the highest, being equal to $1.67 \pm 0.08$. This could be a signal that more particles with lower energy, being modulated by the recurrent solar behavior, were able to reach the Earth. Moreover, the lowest value of $\gamma$ during the recent maximum was $0.96 \pm 0.05$, being $35 \%$ higher in comparison with the value of $\gamma$ during the maximum epoch of SC 23. The middle panel of Figure 1 shows that the rigidity spectrum of the amplitudes of the second harmonic of the GCR recurrence was extremely soft, with the highest value, $\gamma \approx 1.79 \pm 0.09$, being even larger than the $\gamma$ of the first harmonic. The lower panel of Figure 1 illustrates that the lowest value of $\gamma$ for the third harmonics during the recent maximum was $1.02 \pm 0.05$, being almost $60 \%$ higher in comparison with the $\gamma$ during the maximum of SC 23. This lower panel of Figure 1 shows that the rigidity spectrum of the amplitudes of the third harmonic of this variability of the GCR intensity during the recent minimum was soft, with the highest value being $\gamma \approx 1.43 \pm 0.07$. This could be caused by the fact that during the recent minimum epoch, at the end of SC 23, the second and the third harmonics of this variability were indeed enhanced, in addition to the enhancement in the solar-wind and solar-activity parameters (e.g. Modzelewska and Alania, 2013).

To see changes of the spectral index $[\gamma]$ of the amplitudes of the GCR recurrent variation on the background of solar activity and cosmic-ray intensity, the upper panel of Figure 2 displays the 27-day averages of the Oulu NM count rates during the same period of 01 January 2000 to 31 December 2014. One can see a strong compatibility between the NM

Figure 1 Changes of the spectral index $[\gamma]$ of the amplitudes of the first three harmonics of the recurrent variation of the GCR intensity. Upper panel: $\gamma$ of the first harmonic (A27), middle panel: $\gamma$ of the second harmonic (A13.5), and lower panel: $\gamma$ of the third harmonic (A9) calculated using eight neutron monitors (details in Table 1) with different cut-off rigidity during the period 01 January 2000 to 31 December 2014, smoothed over 39 solar rotations.

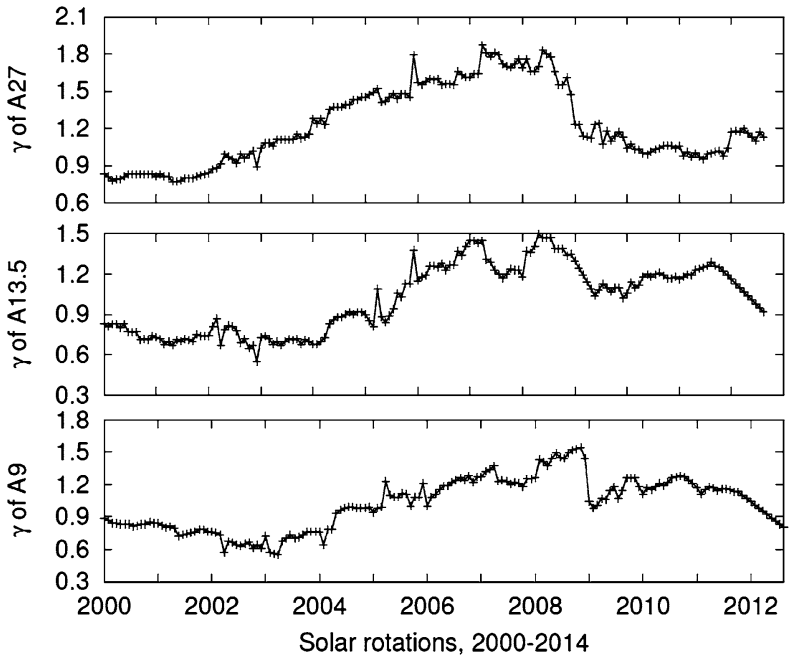


Figure 2 Changes of the cosmic rays measured by the Oulu NM (upper panel), the solar radio flux (middle panel), and the spectral index $[\gamma]$ of the 11-year variation of the GCR intensity calculated using eight neutron monitors (details in Table 1) (lower panel), during the period of 01 January 2000 to 31 December 2014, smoothed over 39 solar rotations.

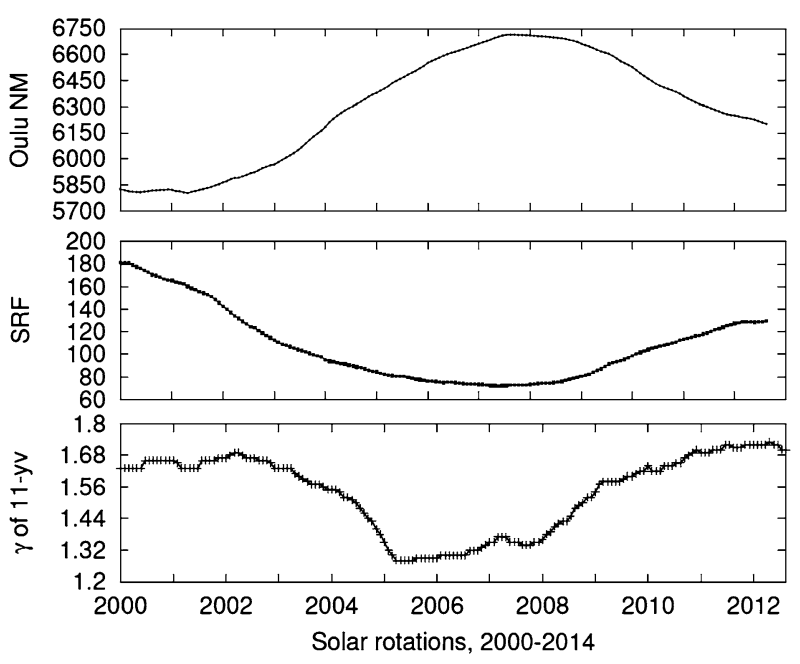

measurements and the spectral index $[\gamma]$. The correlation coefficient between the spectral index $[\gamma]$ and cosmic rays measured by the Oulu station is high: $0.89 \pm 0.02$.

One excellent proxy of solar activity is the solar radio flux (SRF or $F_{10.7}$ index). Its wavelength equals $10.7 \mathrm{~cm}$ and this has been measured continuously since 1947. SRF is derived from the solar atmosphere, high in the chromosphere and low in the solar corona. The middle panel of Figure 2 displays the 27-day averages of SRF during the period of 01 January 2000 to 31 December 2014. There also exists a very clear negative correlation between solar activity illustrated by SRF and the spectral index $[\gamma]$. The anti-correlation between the spectral index $\gamma$ and SRF is high: $-0.89 \pm 0.03$.

Moreover, we have calculated the rigidity spectrum of the 11-year variation of the GCR intensity during the period of 01 January 2000 to 31 December 2014, using the same eight neutron monitors (Table 1). The lower panel of Figure 2 presents the spectral index $[\gamma]$ of the 11-year variation of the GCR intensity, illustrating the fact that there is a strong negative relationship between the changes of the spectral index $[\gamma]$ of the 11-year variation and the spectral index $[\gamma]$ of the recurrent variation of the GCR intensity, with the correlation coefficient being equal to $-0.82 \pm 0.03$. This result is in good agreement with our findings for previous solar-activity cycles (Gil and Alania, 2011).

In order to make a quantitative, not only qualitative, comparison of the rigidity spectrum $[\delta D(R) / D(R)]$ of the amplitudes of the first three harmonics of the recurrent variation of the GCR intensity during the ongoing SC 24 with previous SCs, we have calculated the value of the spectral index $[\gamma]$ of those three harmonics for the period of 01 January 1964 to 31 December 2014 by the method described above, using the Kiel and Rome NMs. Figure 3 shows that the rigidity spectrum of recurrent variation harmonics is, indeed, hard in the maximum and soft in the minimum epochs of solar activity, as was found by Gil and Alania (2010, 2013b). Figure 3 confirms that during the SC 24 the rule, concerning the hardening of the rigidity spectrum of the recurrent variation of the GCR intensity during the solarmaximum epochs, is maintained. Furthermore, Figure 3 endorses the idea that during the ongoing SC 24 values of the spectral index $[\gamma]$ for the first harmonic of this variability of the GCR intensity (upper panel of Figure 3) were at a comparable level as during previous SCs 20-23, but values of $\gamma$ for the amplitudes of the second (middle panel of Figure 3 ) and the third (lower panel of Figure 3 ) harmonics were much higher, from a few up to several dozen percent, than during the previous four solar-activity cycles. 
Figure 3 Changes of the spectral index $[\gamma]$ of the amplitudes of the first three harmonics of the recurrent variation of the GCR intensity. Upper panel: $\gamma$ of the first harmonic (A27), middle panel: $\gamma$ of the second harmonic (A13.5), and lower panel: $\gamma$ of the third harmonic (A9) calculated for the period of 01 January 1964 to 31 December 2014, smoothed over 39 solar rotations.

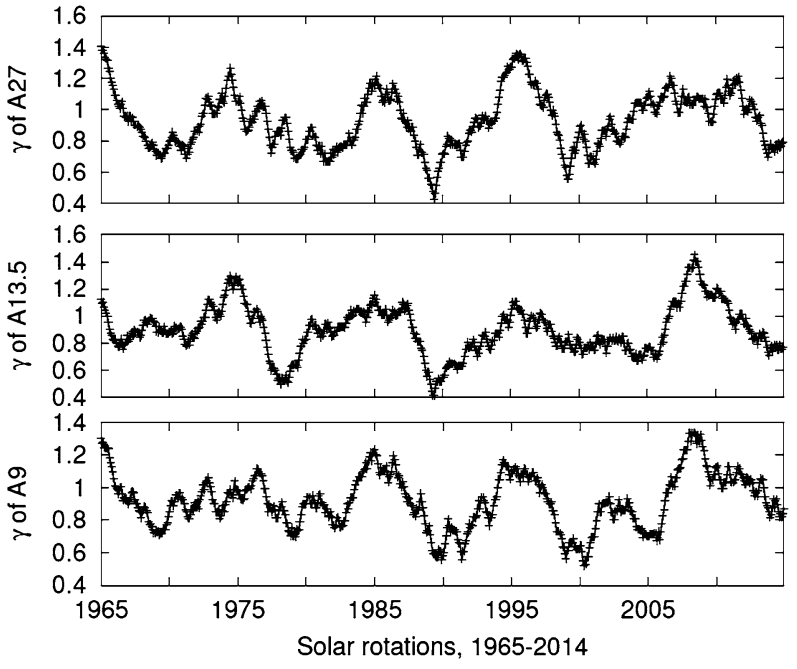

We have explained (Gil and Alania, 2011) the hardening of the rigidity spectrum of the recurrent variation of the GCR intensity in the maximum epochs of SA by the larger average zone of the co-rotating interaction regions (causing the recurrent variation of the GCR intensity) in comparison with the minimum epochs of SA. Hence, a shrinkage of the size of the heliosphere caused by the drop of the solar-wind dynamic pressure during the last Solar Minimum 23/24 (McComas et al., 2008), together with the enhancement of the second and the third harmonics of the recurrent variability effect, could be a significant reason for the exceptional softening of the rigidity spectrum of the second and third harmonics of this recurrence of the GCR intensity during the last Solar Minimum 23/24. Also, the peculiar temporal changes of the coronal-hole structure during SC 23/24 could affect the rigidity spectrum of the recurrent variation.

\section{Conclusions}

i) The energy/rigidity spectrum of the amplitudes of the first three harmonics of the recurrent variation of the galactic cosmic-ray intensity was hard during the maximum epoch of the lasting SC 24 and soft during the recent, unusually prolonged, minimum epoch between SCs 23 and 24. We connected this phenomenon with the changes of the effective size of the modulation region of the recurrent variation of the GCR intensity in different epochs of solar activity.

ii) The energy/rigidity spectrum of the amplitudes of the second and the third harmonics of the 27-day recurrence of the GCR intensity was extremely soft during the last Solar Minimum 23/24 in comparison with the previous four solar minima. We ascribe this phenomenon to the reduction of the heliosphere's extension affected by the decrease of the solar-wind dynamic pressure combined with the enhancement of the second and the third harmonics of the 27-day variation during the Solar Minimum 23/24. In addition, the unusual temporal changes of the coronal-hole structure during SC 23/24 could affect the rigidity spectrum of the recurrent variation.

Acknowledgements We thank the researchers of Apatity, Fort Smith, Jungfraujoch, Kiel, Moscow, Oulu, Potchefstroom, and Rome neutron monitors and http://omniweb.gsfc.nasa.gov for use of their data. 
Open Access This article is distributed under the terms of the Creative Commons Attribution 4.0 International License (http://creativecommons.org/licenses/by/4.0/), which permits unrestricted use, distribution, and reproduction in any medium, provided you give appropriate credit to the original author(s) and the source, provide a link to the Creative Commons license, and indicate if changes were made.

\section{References}

Abramenko, V., Yurchyshyn, V., Linker, J., Mikić, Z., Luhmann, J., Lee, C.O.: 2010, Astrophys. J. $712,813$. ADS. DOI.

Alania, M.V., Wawrzynczak, A.: 2012, Adv. Space Res. 50, 725. ADS. DOI.

Alania, M.V., Modzelewska, R., Wawrzynczak, A.: 2014, J. Geophys. Res. 119, 4164. ADS. DOI.

Aslam, O.P.M., Badruddin: 2015, Solar Phys. 290, 2333. ADS. DOI.

Balogh, A., Hudson, H.S., Petrovay, K., von Steiger, R.: 2014, Space Sci. Rev. 186, 1. ADS. DOI.

Bazilevskaya, G.A., Cliver, E.W., Kovaltsov, G.A., Ling, A.G., Shea, M.A., Smart, D.F., Usoskin, I.G.: 2014, Space Sci. Rev. 186, 409. ADS. DOI.

Clem, J.M., Dorman, L.I.: 2000, Space Sci. Rev. 93, 335. ADS. DOI.

Cliver, E.W., Richardson, I.G., Ling, A.G.: 2013, Space Sci. Rev. 176, 3. ADS. DOI.

Dikpati, M.: 2013, Space Sci. Rev. 176, 279. ADS. DOI.

Dorman, L.I.: 1974, Cosmic Rays: Variations and Space Explorations, North-Holland, Amsterdam. ADS.

Dorman, L.I. (ed.): 2004, Cosmic Rays in the Earth's Atmosphere and Underground, Astrophys. Space Sci. Lib. 303, Kluwer, Dordrecht. ADS.

Dunzlaff, P., Heber, B., Kopp, A., Rother, O., Müller-Mellin, R., Klassen, A., et al.: 2008, Ann. Geophys. 26, 3127. ADS. DOI.

Forbush, S.E.: 1938, J. Geophys. Res. 43, 203. ADS. DOI.

Gil, A., Alania, M.V.: 2008, In: Caballero, R., D’Olivo, J.C., Medina-Tanco, G., Nellen, L., Sánchez, F.A., Valdés-Galicia, J.F. (eds.) Proc. 30th Int. Cosmic Ray Conf. 1, Universidad Nacional Autónoma de México, Mexico City, 601. ADS.

Gil, A., Alania, M.V.: 2010, Adv. Space Res. 45, 429. ADS. DOI.

Gil, A., Alania, M.V.: 2011, J. Atmos. Solar-Terr. Phys. 73, 294. ADS. DOI.

Gil, A., Alania, M.V.: 2013a, Adv. Space Res. 52, 951. ADS. DOI.

Gil, A., Alania, M.V.: 2013b, Solar Phys. 283, 565. ADS. DOI.

Gil, A., Modzelewska, R., Alania, M.V.: 2012, Adv. Space Res. 50, 712. ADS. DOI.

Guo, X., Florinski, V.: 2014, J. Geophys. Res. 119, 2411. ADS. DOI.

Hoeksema, J.T.: 2010, In: Kosovichev, A.G., Andrei, A.H., Rozelot, J.-P. (eds.) IAU Symposium 264, 222. ADS. DOI.

Jian, L.K., Russell, C.T., Luhmann, J.G.: 2011, Solar Phys. 274, 321. ADS. DOI.

Kincaid, D.R., Cheney, E.W.: 2002, Numerical Analysis: Mathematics of Scientific Computing, Pure and applied undergraduate texts, Am. Math. Soc., Providence.

Kirk, M.S., Pesnell, W.D., Young, C.A., Hess Webber, S.A.: 2009, Solar Phys. 257, 99. ADS. DOI.

Kudela, K.: 2013, J. Phys. Conf. Ser. 409, 012017. ADS. DOI.

Mason, G.M., Desai, M.I., Mall, U., Korth, A., Bucik, R., von Rosenvinge, T.T., Simunac, K.D.: 2009, Solar Phys. 256, 393. ADS. DOI.

McComas, D.J., Ebert, R.W., Elliott, H.A., Goldstein, B.E., Gosling, J.T., Schwadron, N.A., Skoug, R.M.: 2008, Geophys. Res. Lett. 35, L18103. ADS. DOI.

Mewaldt, R.A.: 2013, Space Sci. Rev. 176, 365. ADS. DOI.

Mewaldt, R.A., Davis, A.J., Lave, K.A., Leske, R.A., Stone, E.C., Wiedenbeck, M.E., et al.: 2010, Astrophys. J. Lett. 723, L1. ADS. DOI.

Mishev, A.L., Usoskin, I.G., Kovaltsov, G.A.: 2013, J. Geophys. Res. 118, 2783. ADS. DOI.

Modzelewska, R., Alania, M.V.: 2013, Solar Phys. 286, 593. ADS. DOI.

Moraal, H., Stoker, P.H.: 2010, J. Geophys. Res. 115, A12109. ADS. DOI.

Oh, S., Bieber, J.W., Evenson, P., Clem, J., Yi, Y., Kim, Y.: 2013, J. Geophys. Res. 118, 5431. ADS. DOI.

Potgieter, M.S., Vos, E.E., Boezio, M., De Simone, N., Di Felice, V., Formato, V.: 2014, Solar Phys. 289, 391. ADS. DOI.

Richardson, I.G.: 2004, Space Sci. Rev. 111, 267. ADS. DOI.

Sheeley, N.R. Jr.: 2010, In: Cranmer, S.R., Hoeksema, J.T., Kohl, J.L. (eds.) SOHO-23: Understanding a Peculiar Solar Minimum, CS-428, Astron. Soc. Pacific, San Francisco, 3. ADS.

Stozhkov, Y.I., Svirzhevsky, N.S., Bazilevskaya, G.A., Kvashnin, A.N., Makhmutov, V.S., Svirzhevskaya, A.K.: 2009, Adv. Space Res. 44, 1124. ADS. DOI.

Vos, E.E., Potgieter, M.S.: 2015, Astrophys. J. 815, 119. ADS. DOI. 
Vourlidas, A., Howard, R.A., Esfandiari, E., Patsourakos, S., Yashiro, S., Michalek, G.: 2010, Astrophys. J. 722, 1522. ADS. DOI.

Wawrzynczak, A., Alania, M.V.: 2010, Adv. Space Res. 45, 622. ADS. DOI.

Yasue, S., Mori, S., Sakakibara, S., Nagashima, K.: 1982, Coupling Coefficients of Cosmic Ray Daily Variations for NM Stations, Cosmic Ray Res. Lab., Nagoya.

Yeeram, T., Ruffolo, D., Sáiz, A., Kamyan, N., Nutaro, T.: 2014, Astrophys. J. 784, 136. ADS. DOI.

Zhao, L.-L., Qin, G., Zhang, M., Heber, B.: 2014, J. Geophys. Res. 119, 1493. ADS. DOI. 